\title{
Diagnóstico nutricional em cafeeiro conilon orgânico e convencional no Espírito Santo, utilizando o DRIS
}

\author{
Nutrition diagnosis in organic and conventional conilon coffee crop \\ in the State of Espírito Santo, using the DRIS
}

Fábio Luiz Partelli' ${ }^{1}$ Henrique Duarte Vieira ${ }^{2}$ Aureliano Nogueira da Costa $^{3}$

\section{RESUMO}

O objetivo deste trabalho foi realizar o diagnóstico nutricional em cafeeiros conilon cultivados de forma orgânica e convencional na região Norte do Espírito Santo e comparar diferentes índices limiares de diagnóstico para considerar o nutriente como sendo limitante ou excessivo, empregando os teores foliares e produtividade de 40 lavouras sob o cultivo convencional e 56 lavouras sob o cultivo orgânico. Para os cálculos dos índices, utilizou o método DRIS com incorporação da matéria seca. Quatro índices limiares de diagnóstico foram utilizados para considerar o nutriente como sendo limitante. Os nutrientes que ocorreram mais vezes, nas lavouras orgânicas, como sendo deficientes foram $\mathrm{Mn}, \mathrm{P}$, Fe e $\mathrm{Cu}$. Nas lavouras convencionais o $\mathrm{Mn}, \mathrm{N}$ e $P$ foram os nutrientes considerados mais limitantes. Índice de menor valor absoluto afere mais nutrientes como sendo limitante. O primeiro nutriente mais limitante não tem sua posição alterada ao utilizar diferentes índices limiares.

Palavras-chave: Coffea canephora, DRIS, diagnose nutricional.

\section{ABSTRACT}

The objective of this work was to accomplish the nutritional diagnosis in conilon coffee crop cultivated in organic and conventional systems in the North of the Espírito Santo State to compare different limit indexes of diagnose in order to consider the lower and upper nutrient levels by use of foliar contents and the yield of 40 coffee crops under the conventional cultivation and 56 coffee crops under the organic cultivation. The calculations of the indexes were made through the method DRIS including the incorporation of the dry matter. Four indexes of diagnose were used for considering the nutrient as limiting. The nutrients that showed deficiency in the organic coffee crops were $M n, P, F e$ and $\mathrm{Cu}$. In the conventional coffee crop, $\mathrm{Mn}, \mathrm{N}$ and $\mathrm{P}$ were the nutrients limiting. Lower index of absolute value will be able to identify with accuracy the nutrients as limiting. The first limit nutrient does not have its position modified when using different indices thresholds.

Key words: Coffea canephora, DRIS, nutrient diagnosis.

Estudos utilizando a diagnose foliar têm sido muito eficientes, pois a planta é o próprio extrator de nutrientes do solo, possibilitando um diagnóstico nutricional direto e preciso (BEAUFILS, 1973). A correta interpretação de resultados de análises foliares, proporciona informações que favorecem o uso racional de insumos, evita desperdício, melhora o equilíbrio nutricional das plantas e, conseqüentemente, proporciona aumento da produtividade. Portanto, preconiza-se a utilização de métodos que disponibilizam subsídios para um diagnóstico nutricional eficiente e prático, a partir de resultados analíticos das folhas de uma planta e/ou lavoura.

O Sistema Integrado de Diagnose e Recomendação - DRIS, preconizado por BEAUFILS (1973), incorpora o conceito de balanço nutricional ou de equilíbrio entre os minerais nos tecidos das plantas (BALDOCK \& SCHULTE, 1996). Esta técnica se baseia no cálculo de índices para cada nutriente, avaliados em função da relação das razões dos teores de cada elemento com os demais, comparando-os dois a dois,

\footnotetext{
${ }^{1}$ Universidade Estadual do Norte Fluminense Darcy Ribeiro (UENF). Av. Alberto Lamego 2000, Parque Califórnia, $28013-602$, Campos dos Goytacazes, RJ, Brasil. E-mail: partelli@yahoo.com.br

${ }^{2}$ Laboratório de Fitotecnia, Campos dos Goytacazes, UENF, RJ, Brasil.

${ }^{3}$ Instituto Capixaba de Pesquisa, Assistência Técnica e Extensão Rural (INCAPER), Vitória, ES, Brasil
} 
com outras relações consideradas padrões, cuja composição mineral é obtida de uma população de plantas altamente produtivas.

O diagnóstico do estado nutricional através dos índices DRIS fornece também o Índice de Balanço Nutricional - IBN (WADT et al., 1998), que possibilita verificar o equilíbrio nutricional das plantas, indicando, que quanto menor o seu valor, menor é o desequilíbrio nutricional da lavoura amostrada (LEITE, 1993; BALDOCK \& SCHULTE, 1996). O diagnóstico utilizando o DRIS pode verificar limitações de ordem não nutricional, ou seja, lavouras que apresentam baixo IBN e baixa produtividade (LEITE, 1993; WADT et al., 1998). Os diagnósticos elaborados por intermédio do DRIS e com posterior correção do nutriente, proporcionaram maior teor foliar do nutriente e maior produtividade do cafeeiro (ARBOLEDA et al., 1988).

O índice DRIS permite definir o grau de desvio dos nutrientes da amostra, qual sua localização em relação ao estado nutricional, se adequado, em deficiência ou em excesso, indicando a amplitude de cada situação. Sendo assim, pode-se afirmar que um índice de - 6 , usando a constante de sensibilidade igual a $10(z=-0,6)$, indica que o nutriente esta a 0,6 desviospadrão à esquerda da norma, o que se sugere afirmar, baseado na distribuição normal padrão (Teste z), que o estado nutricional de um determinado nutriente está dentro dos 45,2 \% da população ao redor da média, índice em que BATAGLIA et al. (2001) consideraram o nutriente como sendo limitante. Um índice entre -10 e 10 , indica que a amostra está entre os $68,3 \%$ da população centrada em relação à distribuição normal, índice que, segundo REIS Jr (1999), é limiar para um nutriente ser considerado limitante, desde que este seja maior em valor absoluto que o IBNm (Índice de Balanço Nutricional médio).

O objetivo deste trabalho foi realizar o diagnóstico nutricional em cafeeiros conilon cultivados de forma orgânica e convencional na região norte do Espírito Santo e comparar diferentes índices limiares de diagnóstico para considerar o nutriente como sendo limitante (resposta positiva a adubação) ou excessivo.

A coleta das folhas foi realizada em lavouras de café conilon, cultivadas de forma orgânica e convencional, no Norte do Espírito Santo, onde predomina clima tropical, quente e úmido no verão e, inverno seco, com precipitação anual média de 1200 mm e temperatura média de $23^{\circ} \mathrm{C}$. O solo predominante é classificado como Latossolo Vermelho Amarelo Distrófico (ESPÍRITO SANTO, 1994).

Foram selecionadas lavouras representativas da região, sendo 40 sob cultivo convencional (utilização de adubos altamente solúveis e agrotóxicos) e, 56 lavouras sob cultivo orgânico, (certificadas ou em processo de certificação), com no mínimo 30 meses de conversão na colheita (BRASIL, 1999).

Foram realizados levantamentos de vários itens sobre as lavouras, dentre eles, produtividade, insumos utilizados, e outros. Depois, foram realizadas as coletas das folhas de acordo FULLIN \& DADALTO (2001), e quantificados os teores de nutrientes (N, P, $\mathrm{K}, \mathrm{Ca}, \mathrm{Mg}, \mathrm{S}, \mathrm{B}, \mathrm{Cu}, \mathrm{Fe}, \mathrm{Mn}$ e Zn), conforme SILVA (1999).

Na obtenção dos índices dos nutrientes, usaram-se normas específicas, de acordo com a forma de cultivo, utilizando-se as relações e as concentrações dos nutrientes, comparando com as referidas normas, como é feito no M-DRIS (HALLMARK et al., 1987). Para o cálculo da relação normal reduzida dos teores de dois nutrientes, utilizou-se o método de JONES (1981), com a constante de sensibilidade igual a 10. O índice de balanço nutricional - IBN (WADT et al., 1998), consiste no somatório, em valor absoluto de todos os índices envolvidos e o índice de balanço nutricional médio - IBNm é o IBN dividido pelo número de índices envolvidos.

Quatro diferentes índices que proporcionam diagnósticos nutricionais foram interpretados por intermédio do DRIS, nos quais os nutrientes foram considerados limitantes (deficiente ou excessivo), quando os índices de nutrientes foram em valor absoluto maior que 10 e que o IBNm (REIS Jr, 1999), maior em valor absoluto do que sete e que o IBNm, pelo método do potencial de resposta à adubação (PRA) (WADT et al., 1998), onde se considera que o nutriente é provavelmente limitante ou excessivo, quando este apresentar índice em valor absoluto maior que o IBNm e, quando o índice foi, o mais negativo ou mais positivo, nos dois sistemas de cultivo.

Ao verificar o diagnóstico de nutrientes em que se apresentaram como mais negativo ou mais positivo, tanto nas lavouras de café orgânico como nas convencionais (Tabela 1), nota-se que há situações em que alguns nutrientes ocorrem com menor freqüência, num critério do que em outro. No entanto, o primeiro nutriente mais limitante não tem sua posição (maior numero de lavouras) alterada.

Observando os três índices limiares (10, 7 e IBNm) para considerar que um nutriente é limitante, verifica-se que a freqüência dos nutrientes vai aumentando à medida que o valor numérico em valor absoluto do índice do nutriente diminui. Deste modo, de acordo com o índice limiar adotado, o número de lavouras em que o nutriente passa a ser considerado 
Tabela 1 - Número de lavouras de café orgânico e convencional da região norte do Estado do Espírito Santo, em que os nutrientes se apresentaram como sendo limitantes ou excessivo, de acordo com quatro índices limiares ${ }^{(1)}$, utilizando o DRIS com incorporação da matéria seca. Dados entre parênteses são referentes à colocação do nutriente limitante, baseada em número de lavouras.

\begin{tabular}{|c|c|c|c|c|c|c|c|c|}
\hline \multirow{3}{*}{ Nutrientes } & \multicolumn{8}{|c|}{ Índice limiares/números de lavouras que apresentaram os índices } \\
\hline & \multicolumn{4}{|c|}{ Índices negativos (Limitantes) } & \multicolumn{4}{|c|}{ Índices positivos (Excessivos) } \\
\hline & $+\mathrm{NEG}$ & $>10$ & $>7$ & $>\mathrm{IBNm}$ & $+\mathrm{POS}$ & $>10$ & $>7$ & $>\mathrm{IBNm}$ \\
\hline & \multicolumn{8}{|c|}{ Lavouras orgânicas } \\
\hline $\mathrm{N}$ & 7 & 7 & 9 & 14 & 0 & 0 & 0 & 1 \\
\hline $\mathrm{P}$ & 6 & 10 & 13 & 19 & 7 & 6 & 11 & 11 \\
\hline $\mathrm{K}$ & 3 & 2 & 4 & 7 & 13 & 16 & 22 & 26 \\
\hline $\mathrm{Ca}$ & 1 & 2 & 4 & 5 & 0 & 1 & 3 & 3 \\
\hline $\mathrm{Mg}$ & 3 & 4 & 5 & 8 & 4 & 6 & 8 & 10 \\
\hline S & 0 & 3 & 6 & 7 & 1 & 2 & 3 & 7 \\
\hline B & 2 & 3 & 5 & 5 & 8 & 11 & 12 & 18 \\
\hline $\mathrm{Cu}$ & 10 & 12 & 13 & 16 & 7 & 12 & 17 & 19 \\
\hline $\mathrm{Fe}$ & $4\left(5^{\circ}\right)$ & $10\left(3^{\circ}\right.$ ou $\left.4^{\circ}\right)$ & $15\left(2^{\circ}\right)$ & 16 & 5 & 4 & 6 & 8 \\
\hline $\mathrm{Mn}$ & $17\left(1^{\circ}\right)$ & $19\left(1^{\circ}\right)$ & $23\left(1^{\circ}\right)$ & $25\left(1^{\circ}\right)$ & 3 & 3 & 5 & 8 \\
\hline \multirow[t]{2}{*}{$\mathrm{Zn}$} & 3 & 5 & 6 & 8 & 8 & 9 & 11 & 11 \\
\hline & \multicolumn{8}{|c|}{ Lavouras convencionais } \\
\hline $\mathrm{N}$ & 2 & 2 & 7 & 11 & 1 & 0 & 1 & 3 \\
\hline $\mathrm{P}$ & 5 & 4 & 10 & 10 & 2 & 2 & 3 & 4 \\
\hline $\mathrm{K}$ & 2 & 0 & 2 & 3 & 2 & 1 & 5 & 7 \\
\hline $\mathrm{Ca}$ & 1 & 1 & 3 & 8 & 3 & 4 & 5 & 7 \\
\hline $\mathrm{Mg}$ & 4 & 3 & 7 & 8 & 1 & 2 & 3 & 5 \\
\hline $\mathrm{S}$ & 4 & 3 & 3 & 8 & 1 & 1 & 3 & 4 \\
\hline B & 4 & 3 & 5 & 8 & 5 & 3 & 9 & 10 \\
\hline $\mathrm{Cu}$ & 5 & 6 & 7 & 9 & 9 & 10 & 15 & 17 \\
\hline $\mathrm{Fe}$ & 2 & 2 & 5 & 6 & 5 & 6 & 9 & 9 \\
\hline $\mathrm{Mn}$ & 9 & 8 & 11 & 13 & 9 & 8 & 12 & 12 \\
\hline $\mathrm{Zn}$ & 2 & 2 & 6 & 8 & 2 & 4 & 5 & 8 \\
\hline
\end{tabular}

${ }^{(1)}$ Nutriente em que o índice foi mais negativo (+NEG), mais positivo (+POS), Índice de nutriente maior que 10 em valor absoluto e maior que o IBNm (>10), Índice de nutriente maior que sete em valor absoluto e, maior que o IBNm (>7) e Índice de nutriente maior que o IBNm em valor absoluto (>IBNm).

deficiente ou excessivo é alterado nos dois grupos de lavouras (Tabela 1). Estes relatos indicam que o fato de utilizar um determinado índice limiar para aferir deficiência ou excesso de um nutriente em uma planta e/ou lavoura, vai depender do rigor preestabelecido, ou seja, menor índice do nutriente em valor absoluto afere, excesso ou deficiência em maior número de lavouras (maior freqüência), assim, num diagnóstico geral de uma região indicará mais nutrientes como sendo limitantes e excessivos, e maior índice limiar, em valor absoluto, indicará menos nutrientes como limitantes (ocorrência em menor número de lavouras). Portanto, é plausível que se use como índice limiar; índices menores, quando se tem elevada tecnologia, alta produtividade pendente, ou quando se deseja obter elevada produtividade, isto se as limitações estiverem relacionadas à nutrição. Sendo assim, o método PRA, segue esta tendência, pois verifica-se, em alguns trabalhos, uma correlação negativa entre a produtividade e o IBN (LEITE, 1993), entretanto, há casos de lavouras com baixo IBN e com baixa produtividade, portanto, não é regra adotar este método.

Ao verificar o número de lavouras em que os nutrientes apresentam-se como sendo o mais deficiente (+NEG) nas lavouras orgânicas, percebese, por exemplo, que o ferro (Fe) aparece em quinta colocação em número de lavouras (quatro lavouras) como sendo o elemento mais limitante (Tabela 1 , parentese); já quando o índice que considera o nutriente limitante foi o índice limiar menor que -10 e maior que o IBNm em valor absoluto, o Fe passou a aparecer em terceira ou quarta colocação em número de lavouras (10 lavouras). Ao utilizar o índice limiar igual ou menor que -7 (quando o nutriente está dentro dos 50 \% da população ao redor da média) e maior que

Ciência Rural, v.35, n.6, nov-dez, 2005. 
o IBNm em valor absoluto, o Fe passou a ficar em segunda colocação em número de lavouras (15 lavouras) como sendo um nutriente, possivelmente, limitante, demonstrando que, à medida que o índice limiar é alterado, a ordem dos nutrientes deficientes e excessivos muda. No entanto, o primeiro nutriente mais limitante não tem sua posição alterada com a mudança do índice limiar (maior número de lavouras), conforme pode ser verificado nas tabela 1 , (parêntese) e 2 , para o nutriente Mn, nos cultivos orgânico e convencional.

A média do IBN das lavouras orgânicas e convencionais que apresentaram produtividade igual e superior a 40 sacas beneficiadas de $60 \mathrm{~kg}$ por hectare, foram respectivamente, 63,06 e 66,65, vindo a indicar que o equilíbrio nutricional dos dois grupos de lavouras foi próximo. Entretanto, a média do IBN das lavouras orgânicas com produtividade inferior a 40 sacas por hectare foi de 104,99, sugerindo, que um dos principais motivos da baixa produtividade, de muitas destas lavouras, pode estar relacionado à desequilíbrios nutricionais.

Ao realizar o diagnóstico nas lavouras orgânicas e convencionais (tabela 1), observa-se que o nutriente que ocorreu em maior número de lavouras como limitante foi o Mn. LEITE (1993) e COSTA et al. (2000) também relataram que o Mn apresentou-se, em situações específicas, como um nutriente problemático por deficiência para o cafeeiro conilon no Espírito Santo. Este grande número de lavouras apresentando o Mn como limitante, possivelmente, está relacionado ao alto $\mathrm{pH}$ em determinados solos, fato este que, segundo MARSCHNER (1995), deixa o Mn menos disponível para as plantas. Provavelmente, o alto $\mathrm{pH}$ ocorreu devido o excesso de aplicação de calcário e falta de incorporação do mesmo, pois, ao verificar o histórico destas lavouras, constatou-se que havia solos que receberam calcário sem uma prévia análise de solo e em muitos casos a dose recomendada foi para camada de 0 a 20 centímetros do solo e este não foi devidamente incorporado ao solo.

O Mn também ocorreu em grande número de lavouras convencionais como sendo o nutriente que apresentou índice mais positivo, fato também relatado por LEITE (1993). Isso, possivelmente, pode está associado à solos ácidos, ocasionado pelo uso contínuo de doses elevadas de adubos nitrogenados acidificantes, o que levaria a uma grande disponibilidade de Mn, (MARSCHNER, 1995).

Outros nutrientes como o $\mathrm{Cu}, \mathrm{P}$, e $\mathrm{N}$ também se apresentaram como possíveis nutrientes limitantes em grande número, nos dois grupos de lavouras e o Fe nas lavouras orgânicas. Esses resulatados corroboram os observados por LEITE (1993) em que o
$\mathrm{Cu}$ foi o nutriente que em mais lavouras, depois do Mn se apresentou como nutriente mais limitante e, com COSTA et al. (2000) em que o P e o Fe apresentaram-se em grande número de lavouras como sendo nutriente limitante nas lavouras de conilon. A deficiência desses nutrientes, possivelmente, deve estar relacionada à baixa concentração dos mesmos nos solo, por origem e/ou por cultivos consecutivos, e em muitas lavouras os nutrientes não foram adicionados, ou foram adicionados em quantidades insuficientes. Estes solos são originalmente pobres quimicamente e devido a um manejo inadequado são encontrados solos com baixo pH e outros com alto, o que pode ter influenciado negativamente na absorção destes nutrientes (MARSCHNER, 1995).

Conclui-se que os nutrientes que ocorreram com maior freqüência nas lavouras orgânicas como deficientes foram $\mathrm{Mn}, \mathrm{P}, \mathrm{Fe}$ e $\mathrm{Cu}$, e nas lavouras convencionais foram o Mn, N e P. Índice menor em valor absoluto afere mais nutrientes como sendo limitantes e, altera o número de lavouras que apresentam um determinado nutriente como sendo limitante nos dois sistemas de cultivos. O primeiro nutriente mais limitante não tem sua posição alterada ao utilizar diferentes índices limiares. O IBN das lavouras orgânicas e convencionais foi próximo.

\section{AGRADECIMENTOS}

Os autores agradecem à Universidade Estadual do Norte Fluminense Darcy Ribeiro - UENF, Fundação de Amparo à Pesquisa do Estado do Rio de Janeiro - FAPERJ, MIBASA Mineração Barreto, Instituto Capixaba de Pesquisa Assistência Técnica e Extensão Rural - INCAPER, BIOSYSTEMS e aos agricultores.

\section{REFERÊNCIAS}

ARBOLEDA, C.V. et al. Sistema integrado de recomendación y diagnosis: uma alternativa para la interpretación del análisis foliar en café. Agronomia Colombiana, Bogotá, v.5, p.17-30, 1988.

BATAGLIA, O.C. et al. Monitoramento nutricional de cafezais usando o critério de faixas de suficiência e o DRIS. In: SIMPÓSIO BRASILEIRO DE PESQUISA DOS CAFÉS DO BRASIL, 2., 2001, Brasilia, DF. Anais... Brasília: Embrapa Café, 2001. p.2576-2581.

BALDOCK, J.O.; SCHULTE, E.E. Plant analysis with standardized scores combines DRIS and sufficiency range approaches for corn. Agronomy Journal, Madison, v.88, p.448-456, 1996.

BEAUFILS, E.R. Diagnosis and recommendation integrated system (DRIS). A general scheme of experimentation and calibration based on principles developed from research in plant nutrition. South Africa: University of Natal, Pietermaritzburg. 1973. 132p. (Soil Science Bulletin, 1). 
BRASIL Ministério da Agricultura e do Abastecimento. Instrução Normativa n. 7, de 17 de maio de 1999. Dispõe sobre normas para a produção de produtos orgânicos vegetais e animais. Diário Oficial da República Federativa do Brasil, Poder Executivo, Brasília, DF, 19 de maio 1999. Seção 1. 1999.

COSTA, A.N. da., et al. Levantamento nutricional do cafeeiro conilon pelo DRIS, no Espírito Santo. In: SIMPÓSIO BRASILEIRO DE PESQUISA DOS CAFÉS DO BRASIL, 2000, Poços de Caldas, MG. Anais... Poços de Caldas: Embrapa Café, 2000. p.1333-1335.

ESPÍRITO SANTO (Estado). Secretaria de Estado de Ações Estratégicas e Planejamento. Informações municipais do Estado do Espírito Santo 1994. Vitória: Departamento Estadual de Estatística, 1994. V.1, 803p.

FULLIN, E.A.; DADALTO, G.G. Avaliação da fertilidade do solo e do estado nutricional das plantas. In: DADALTO, G.G.; FULLIN, E.A. Manual de recomendação de calagem e adubação para o Estado do Espírito Santo. (quarta aproximação). Vitória: SEEA \& INCAPER, 2001. p.21-55.

HALLMARK, W.B. et al. Comparison of two DRIS methods for diagnosing nutrient deficiencies. Journal of Fertizers Issues, Manchester, v.4, p.151-158, 1987.

JONES, W.W. Proposed modifications of the diagnosis and recommendation integrated system (DRIS) for interpreting plant analyses. Communications in Soil Science and Plant Analysis, New York, v.12, n.8, p.785794, 1981.

LEITE, R.A. Avaliação do estado nutricional do cafeeiro conilon no Estado do Espírito Santo utilizando diferentes métodos de interpretação de análise foliar. 1993. 87f. Tese (Doutorado em Solos e Nutrição de Plantas) - Curso de Pós-graduação em Solos e Nutrição de Plantas, Universidade Federal de Viçosa.

MARSCHNER, H. Mineral nutrition of higher plants. London: Academic, 1995. 889p.

REIS Jr, R. dos A. Diagnose nutricional da cana-deaçúcar com o uso do sistema integrado de diagnose e recomendação (DRIS). 1999. 141f. Tese (Doutorado em Produção Vegetal) - Curso de Pós-graduação em Produção Vegetal, Universidade Estadual do Norte Fluminense.

SILVA, F.C. da. (org). Manual de análises químicas de solos, plantas e fertilizantes. Brasília: Embrapa, 1999. $370 \mathrm{p}$.

WADT, P.G.S. et al. Três métodos de cálculo do Dris para avaliar o potencial de resposta à adubação de árvores de eucalipto. Revista Brasileira de Ciência do Solo, Viçosa, v.22, p.651-660, 1998. 